Revista Brasileira de Agricultura Irrigada v.14, no .2, p. 3960 - 3967, 2020

ISSN 1982-7679 (On-line)

Fortaleza, CE, INOVAGRI - http://www.inovagri.org.br

DOI: $10.7127 /$ rbai.v14n101146

Protocolo 1146.20 - 20/04/2020 Aprovado em 18/06/2020

\title{
COEFICIENTE DE CULTURA POR LISÍMETRIA DA VARIEDADE DE SORGO FORRAGEIRO SF - 15 (SORGHUM BICOLOR L. MOENCH)
}

\author{
Vinícius Auer Xavier Schwenck ${ }^{1}$, Lucas da Silva Queiroz ${ }^{2}$, Andre Dalla Bernardina Garcia ${ }^{3}$, Kevilin
} De Jesus Leite ${ }^{4}$, Stella Arndt ${ }^{5}$, Ednaldo Miranda de Oliveira ${ }^{6}$

\begin{abstract}
RESUMO
O sorgo forrageiro (Sorghum bicolor L. Moench), tem se apresentado como uma boa alternativa na produção de forragens com aptidão para corte e silagem, dada sua característica de maior adaptabilidade a regiões com regime de chuva irregular, solos rasos e de baixa fertilidade. A determinação das necessidades hídricas das culturas é usualmente estimada com base na evapotranspiração de referência de um local, coeficiente hídrico específico da espécie estudada, e com isso é correlacionado à lâmina necessária para suprir as necessidades hídricas da planta num local. A realização deste trabalho teve como objetivo determinar os valores de coeficientes de cultura em todos os estádios fenológicos do sorgo forrageiro utilizando lisímetros de drenagem. $\mathrm{O}$ experimento foi conduzido numa área experimental localizada nas coordenadas geográficas de $19^{\circ} 48^{\prime} 17^{\prime}$ ' $\mathrm{S}, 40^{\circ}$ 40'35' O e altitude de $136 \mathrm{~m}$. Foram utilizados três lisímetros de drenagem com dimensões de 1,10 $\mathrm{m}$ de largura, 1,60 $\mathrm{m}$ de comprimento e $0,70 \mathrm{~m}$ de profundidade. Uma estação automática foi utilizada para estimar a evapotranspiração de referência pelo método padrão de Penman-Monteith. A evapotranspiração de referência e a evapotranspiração total da cultura do sorgo, para um ciclo de 92 dias, foram $355,6 \mathrm{~mm}$ e $492,9 \mathrm{~mm}$, respectivamente. Os coeficientes de cultivo obtidos para os diferentes estádios fenológicos da cultura foram de 0,7 (Inicial), 1,2 (Crescimento), 1,8 (Floração) e 1,2 (Maturação).
\end{abstract}

Palavras-chave: Agrometeorologia, nutrição animal, balanço hídrico.

\footnotetext{
${ }^{1}$ Graduando em Agronomia, Instituto Federal do Espírito Santo, Santa Teresa, ES, Rodovia ES-080, Km 93 - São João de Petrópolis, CEP: 29660-000. E-mail: viniciusschwenck@gmail.com

${ }^{2}$ Engenheiro Agrônomo, Instituto Federal do Espírito Santo, Santa Teresa, ES, Rodovia ES-080, Km 93 - São João de Petrópolis, CEP: 29660-000. E-mail: lucassqueiroz3@gmail.com

${ }^{3}$ Mestrando em Produção Vegetal, Universidade Estadual do Norte Fluminense/UENF, Campos dos Goytacazes, RJ, Av. Alberto Lamego nº 2000, CEP: 28013-602, e-mail: andredallabg@ outlook.com

${ }^{4}$ Graduando em Agronomia, Instituto Federal do Espírito Santo, Santa Teresa, ES, Rodovia ES-080, Km 93 - São João de Petrópolis, CEP: 29660-000. E-mail: kevilin.leite@ gmail.com

${ }^{5}$ Graduando em Agronomia, Instituto Federal do Espírito Santo, Santa Teresa, ES, Rodovia ES-080, Km 93 - São João de Petrópolis, CEP: 29660-000. E-mail: stellaarndt7@gmail.com

${ }^{6}$ Doutor em Recursos Hídricos e Ambientais e Docente, Instituto Federal do Espírito Santo, Santa Teresa, ES, Rodovia ES-080, Km 93 - São João de Petrópolis, CEP: 29660-000, e-mail: ednaldo.oliveira@ifes.edu.br
} 


\begin{abstract}
Forage sorghum (Sorghum bicolor L. Moench), has been presented as a good alternative in the production of forages with aptitude for cutting and silage, given its characteristic of greater adaptability to regions with irregular rain regime, shallow soils and low fertility. The determination of the water requirements of the crops is usually estimated based on the reference evapotranspiration of a location, the specific water coefficient of the studied species, and with this the correlation of the blade needed to supply the water needs of the plant in a location. The purpose of this work was to determine the values of culture coefficients in all phenological stages of forage sorghum using drainage lysimeters. The experiment was carried out in an experimental area located at the geographical coordinates of $19^{\circ} 48^{\prime} 17^{\prime}$ ' S, 40 40'35' $\mathrm{O}$ and an altitude of $136 \mathrm{~m}$. Three drainage lysimeters with dimensions of $1.10 \mathrm{~m}$ wide, $1.60 \mathrm{~m}$ long and $0.70 \mathrm{~m}$ deep were used. An automatic station was used to estimate the reference evapotranspiration using the standard Penman-Monteith method. The reference evapotranspiration and total evapotranspiration of the sorghum culture, for a cycle of 92 days, were $355.6 \mathrm{~mm}$ and $492.9 \mathrm{~mm}$, respectively. The cultivation coefficients obtained for the different phenological stages of the culture were 0.7 (Initial), 1.2 (Growth), 1.8 (Flowering) and 1.2 (Maturation).
\end{abstract}

Keywords: Agrometeorology, animal nutrition, water balance.

\section{INTRODUÇÃO}

A escassez de alimentos, principalmente a forragem fornecida na alimentação dos ruminantes, que é agravada no período seco, e o baixo valor nutritivo da pastagem natural comprometem o desenvolvimento dos animais, acarretando em queda de produtividade e comprometendo a produção de leite e carne, levando a uma dependência de volumosos conservados, como farelo de milho e soja, por parte dos produtores (AMORIM et al., 2017).

De acordo com Queiroz et al. (2015) o sorgo forrageiro (Sorghum bicolor (L.) Moench), espécie vegetal de elevado potencial de matéria seca, e que constitui a alimentação do rebanho, tem se apresentado como uma boa alternativa na produção de forragens com aptidão para corte e silagem, dada sua característica de maior adaptabilidade a regiões com regime de chuva irregular, solos rasos e de baixa fertilidade.

Estudos que apresentam a importância da água para forrageiras, pouco vem sendo realizados no Brasil (OLIVEIRA et al., 2015; DANTAS et al., 2016). Nesse sentido, faz-se necessário a execução de trabalhos que mostrem o comportamento, produção e desenvolvimento dessas culturas quando em excesso ou escassez hídrica. Fato é que a água é absorvida na solução do solo pelas plantas através de seu sistema radicular, deste modo, o uso de ferramentas de manejo da irrigação que auxilie no controle da lâmina reposta no sistema solo-planta é fundamental para tornar o sistema produtivo sustentável.

$\mathrm{O}$ uso de estações meteorológicas automáticas para determinação das variáveis climáticas vem sendo difundido, haja vista a qualidade dos dados gerados, que dão melhor respaldo ao agricultor na aplicação de água na lavoura via sistema de irrigação, estimando através das variáveis meteorológicas a ETo local (GARCIA et al., 2019). Entretanto, o custo na aquisição desse equipamento, torna-o pouco acessível a sistemas de produção de caráter familiar, como é o caso da maioria das propriedades do estado do Espírito Santo.

Segundo Kobayashi et al. (2018), outro componente do estudo das exigências hídricas nas espécies cultivadas é o Kc, obtido pela razão entre a evapotranspiração da cultura trabalhada e a de referência (dados de uma cultura hipotética), correlacionando-as à fase de desenvolvimento e às condições climáticas do local de estudo, podendo ser adotado em outras localidades desde que compartilhe dos mesmos padrões climáticos.

A distribuição do $\mathrm{Kc}$ durante o ciclo produtivo, é denominado "curva da cultura", e 
representa o efeito integrado da mudança de área foliar, do grau de cobertura, da altura da planta, da resistência do dossel a perda de água e do albedo sobre a ETc em relação a ETo. Por isso quanto maior o valor de $\mathrm{Kc}$, maior a necessidade de irrigação, e quanto menor o índice, menor também deverá ser a aplicação de água (CONCEIÇÃO et al, 2016).

Nesse sentido, a lisimetria é utilizada com o objetivo de se conhecer alguns termos do balanço hídrico do volume amostrado através de um reservatório impermeável contendo um volume de solo. Sendo assim, o uso de lisímetros pelo método de pesagem e de drenagem são indicados.

Para se obter medições mais precisas da evapotranspiração (ET), é recomendado o uso do lisímetro de pesagem, considerado o método mais preciso para medida da ET. No entanto, desde que sejam respeitadas as normas básicas de construção, calibração e metodologia de leitura, os lisímetros de drenagem são apresentados como métodos alternativos (MARQUES et al., 2015; ALVES et al., 2017).

Diante deste cenário, a realização deste trabalho teve como objetivo determinar os valores de coeficientes de cultura $(\mathrm{Kc})$ em todos os estádios fenológicos do sorgo forrageiro utilizando lisímetros de drenagem.

\section{MATERIAIS E MÉTODOS}

O experimento foi conduzido numa área cultivada com sorgo (Sorghum bicolor (L.) Moench), variedade sorgo forrageiro SF-15 em condições de campo no Instituto Federal de Educação, Ciência e Tecnologia do Espírito Santo (IFES) - campus Santa Teresa, localizado nas coordenadas geográficas de $19^{\circ} 48^{\prime} 17^{\prime \prime}$ latitude $\mathrm{S}, 40^{\circ} 40^{\prime} 35^{\prime}$ ' longitude $\mathrm{O}$ e altitude de 136 m. A construção e instalação da estação lisimétrica foi realizada ao lado da estação meteorológica, com caixas de cimento amianto de $1.000 \mathrm{~L}(1,10 \mathrm{~m}$ de largura, $1,60 \mathrm{~m}$ de comprimento e $0,70 \mathrm{~m}$ de profundidade), e o sistema de drenagem construído a partir de tubos PVC e instalado na parte inferior das caixas, preenchidas com uma camada de brita zero, sobreposta por outra de areia, seguida de solo destorroado e peneirado, proveniente de Latossolo Vermelho-Amarelo. Amostras do solo dos lisímetros foram submetidas a análises química e físico-hídrica para determinação da densidade, textura e retenção de água em laboratório do próprio campus. A correção da acidez do solo e a sua fertilização foram realizadas de acordo com os resultados da análise química e de acordo com as exigências nutricionais da cultura do sorgo plantado dentro dos lisímetros. As adubações de cobertura foram feitas de acordo com recomendações técnicas indicadas para a cultura.

Os lisímetros foram cultivados com sorgo no espaçamento de $0,80 \mathrm{~m}$ entre linhas e 0,07 $\mathrm{m}$ entre plantas, e no espaçamento do lado externo foram considerados o mesmo padrão de plantio, margeando os lisímetros com o objetivo de neutralizar o efeito oásis (bordadura). $\mathrm{O}$ plantio foi realizado manualmente, e o material genético utilizado foi a variedade sorgo forrageiro SF-15.

Ao longo do desenvolvimento do experimento foram realizados tratos culturais necessários, seguindo recomendações técnicas exigidas pela cultura. A evapotranspiração do sorgo nos lisímetros foi determinada por meio do balanço hídrico, desconsiderando a variação do armazenamento, de acordo com a Equação 1:

$$
E T c=P+I-D
$$

Em que: ETc é a Evapotranspiração da cultura (mm dia $\left.{ }^{-1}\right)$, "P" representa a precipitação pluvial no lisímetro (mm), "I" á a lâmina de água aplicada por irrigação (mm) e D indica a lâmina de água drenada (mm).

A evapotranspiração de referência (ETo) foi obtida com o uso do programa computacional Ref ET (ALLEN, 2000), aplicando-se a equação de PenmanMonteith (ALLEN et al., 1998) (Equação 2) subsidiada com os dados obtidos da estação meteorológica instalada no próprio campo experimental. 
$\mathrm{ET}_{0}=\frac{0.408 \Delta\left(\mathrm{R}_{\mathrm{n}}-\mathrm{G}\right)+\gamma \frac{900}{\mathrm{~T}+273} \mathrm{U}_{2}\left(\mathrm{e}_{\mathrm{s}}-\mathrm{e}_{\mathrm{a}}\right)}{\Delta+\gamma\left(1+0.34 \mathrm{U}_{2}\right)}$

Em que: Rn - Radiação líquida, em MJ m-2 s-1; G - Densidade do fluxo de calor no solo, em MJ m-2 s-1; T - Temperatura média diária do ar, em ${ }^{\circ} \mathrm{C} ; \gamma$ - Constante psicrométrica, em $\mathrm{kPa}$ OC-1; $\Delta$ - Tangente à curva de pressão de saturação de vapor em função da temperatura do ar, em kPa OC-1; U2 - Velocidade média diária do vento a 2 metros de altura, em m s-1; ea - Pressão de vapor de saturação, média diária, em kPa; es - Pressão de vapor de saturação, média diária, à temperatura do ponto de orvalho, em $\mathrm{kPa}$.As irrigações e a coleta da água percolada nos lisímetros foram realizadas com frequência de 24 horas ao longo de todo o período experimental.

Os coeficientes de cultura $\mathrm{KC}$ ), que relacionam a evapotranspiração de determinada cultura com a evapotranspiração de referência $m$ condições ótimas de umidade, fertilidade e sanidade, em seus diferentes estádios de seu desenvolvimento foram determinados pela equação 3:
$K c=\frac{E T c}{E T o}$

Em que: Kc é o coeficiente da cultura, ETc é a evapotranspiração da cultura $\left(\mathrm{mm} \mathrm{dia}^{-1}\right)$ e ETo representa a evapotranspiração de referência $\left(\mathrm{mm} \mathrm{dia}^{-1}\right)$. Os valores de $\mathrm{K}_{\mathrm{C}}$ foram determinados diariamente e agrupados no intervalo de dias de cada estádio de desenvolvimento, sendo que o valor médio para cada fase representa o $\mathrm{K}_{\mathrm{C}}$ para as condições daquela cultura e para a região onde foi determinada. Foram realizadas determinações diárias dos valores de evapotranspiração da cultura, evapotranspiração de referência e a estimativa do $\mathrm{K}_{\mathrm{C}}$ nos 3 lisímetros de drenagem. Cada lisímetro constituiu uma repetição.

\section{RESULTADOS E DISCUSSÃO}

Por meio de coleta de dados da estação meteorológica foi possível obter os valores de temperatura máxima e mínima e de umidade relativa (Figura 1), que são variáveis necessárias para calcular a evapotranspiração de referência $\left(\mathrm{ET}_{\mathrm{O}}\right)$.

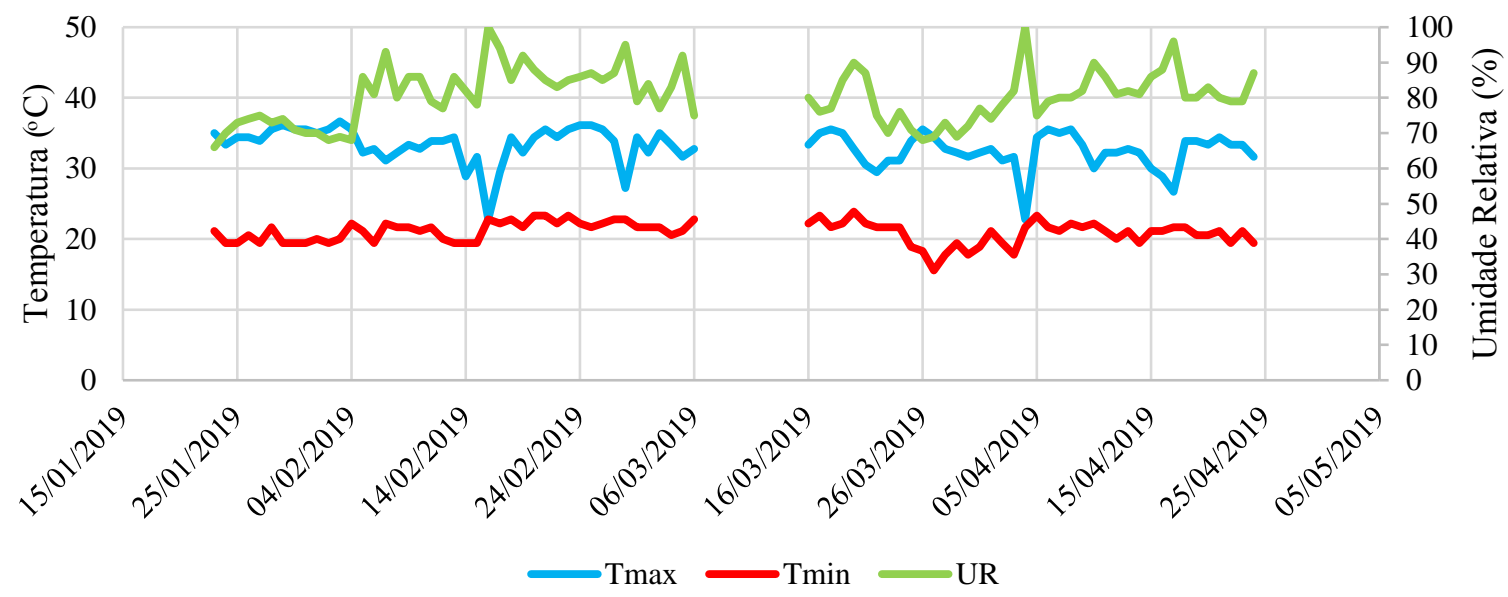

Figura 1. Dados meteorológicos obtidos na estação automática durante o período experimental.

Por meio da Figura 1 é possível notar que houve variações na temperatura máxima e mínima e na umidade relativa com o passar do tempo. As médias das temperaturas máxima e mínima foram $36,7{ }^{\circ} \mathrm{C}$ e $15,6{ }^{\circ} \mathrm{C}$, respectivamente no período de condução do experimento. A umidade relativa do ar média foi $80,5 \%$, não apresentando grandes variações. No período de 07 à 15 de março de 2019 não foi possível a obtenção de dados, pois a estação meteorológica apresentou uma falha e não armazenou os valores das variáveis 
meteorológicas. De posse dos dados obtidos diariamente de entrada e saída de água nos lisímetros foi possível a obtenção da evapotranspiração da cultura (ETc) e com os dados da estação meteorológica foram obtidos os resultados de evapotranspiração de referência (ETo) utilizando o método de Penman-Monteith. As evapotranspirações da cultura e de referência estão apresentadas na Figura 2.

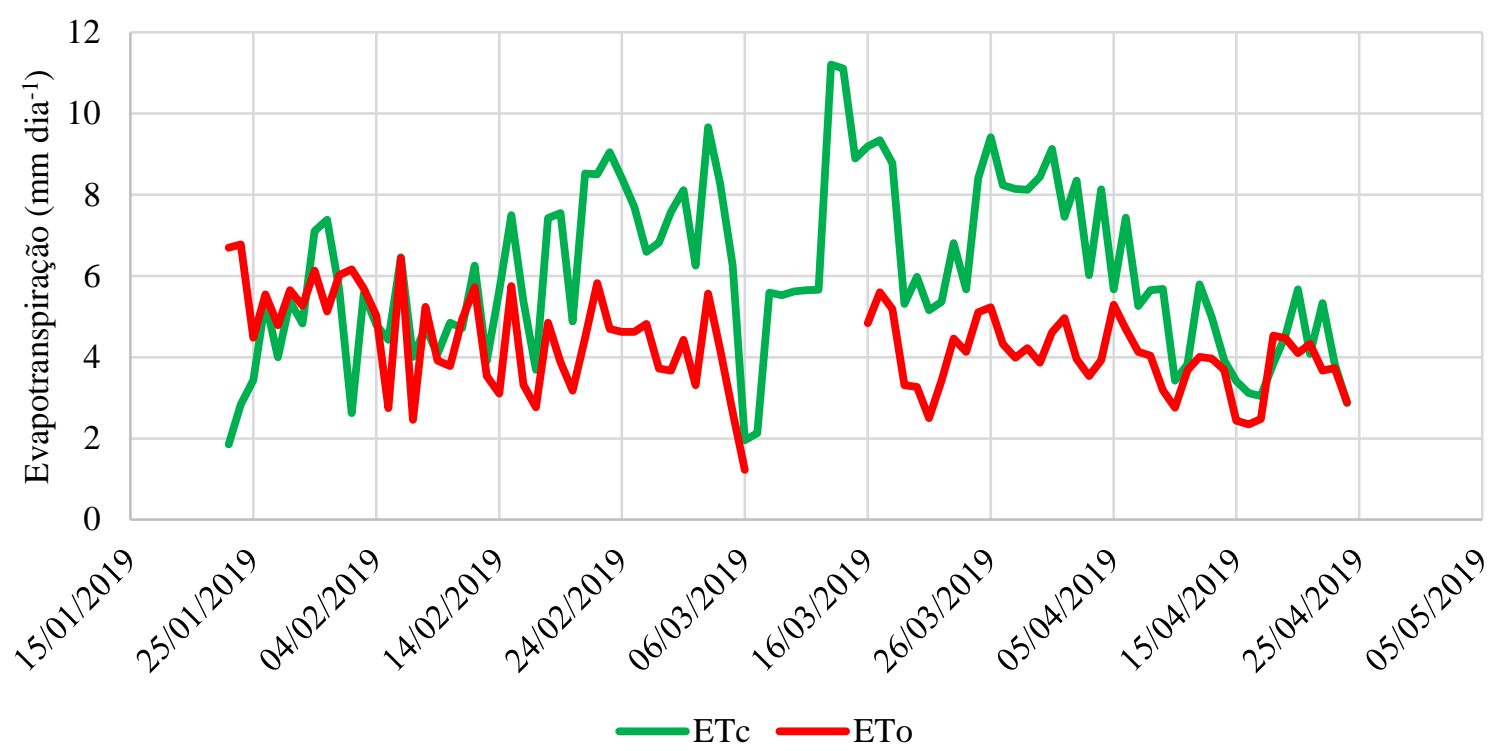

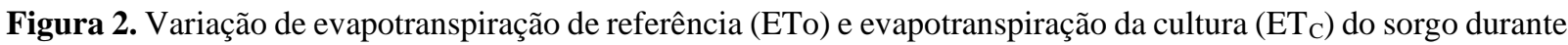
os meses de janeiro a abril de 2019.

Pela análise gráfica é possível perceber que as evapotranspirações da cultura e de referência alternam o maior valor entre elas, ou seja, ora ETo é maior que ETc, e, ora ETc é maior que ETo. Isso é explicado pelo fato de que ETc é o valor que está relacionado a evapotranspiração da cultura, portanto, quando a cultura se apresenta em estágio inicial ou fase final de desenvolvimento a ETc será menor que ETo, devido à baixa transpiração da área de superfície foliar, enquanto quando em estágio de desenvolvimento do sorgo a evapotranspiração da cultura ultrapassa os valores de ETo, pois há grande transpiração foliar, conforme elucidado por alguns autores (MEHTA e PANDEY, 2015; GIRARDI et al, 2016).

O valor máximo obtido para evapotranspiração de referência foi 6,78 e o mínimo de $1,22 \mathrm{~mm} \mathrm{dia}^{-1}$. Isso é explicado devido a mudanças nas condições do clima, que ocorrem naturalmente com o passar do tempo.
Os valores de evapotranspiração da cultura máximo e mínimo correspondem a 9,66 e $1,85 \mathrm{~mm} \mathrm{dia}^{-1}$, respectivamente, para $\mathrm{o}$ período de condução do experimento. No início do desenvolvimento da cultura a $\mathrm{ET}_{\mathrm{C}}$ tende a ser menor por conta da menor demanda hídrica pela planta. Para a planta atingir o desenvolvimento vegetativo pleno demanda nutrientes e água em maior quantidade. Percebe-se este comportamento no período em que a $\mathrm{ET}_{\mathrm{C}}$ é maior que a $\mathrm{ET}_{\mathrm{O}}$. No estadio final da planta, quando ocorre sua senescência, a planta necessita de menos recursos e, portanto, utiliza menos água.

Fonsêca Neto (2014), em trabalho realizado com sorgo, obteve valores de 7,84 $\mathrm{mm} \mathrm{dia}{ }^{-1}$ e de $3,99 \mathrm{~mm} \operatorname{dia}^{-1}$ para evapotranspiração de referência $\left(\mathrm{ET}_{\mathrm{O}}\right)$ e para Evapotranspiração de cultura $\left(\mathrm{ET}_{\mathrm{C}}\right)$, respectivamente, numa variação de 1,72 a 7,84 $\mathrm{mm} \mathrm{dia}{ }^{-1}$ no município de Ipanguaçu/RN. Os valores determinados neste trabalho são 
semelhantes aos encontrados no presente estudo, o que mostra a similaridade da necessidade de água para esta cultura nas diferentes regiões. $\mathrm{Na}$ Figura 3, estão apresentados os coeficientes de cultura para o sorgo entre os meses de janeiro e abril. Ressalta-se que entre 07 e 15 de março não há valores de Kc, pois devido à falha no armazenamento dos dados meteorológicos, não foi possível calcular a ETo.

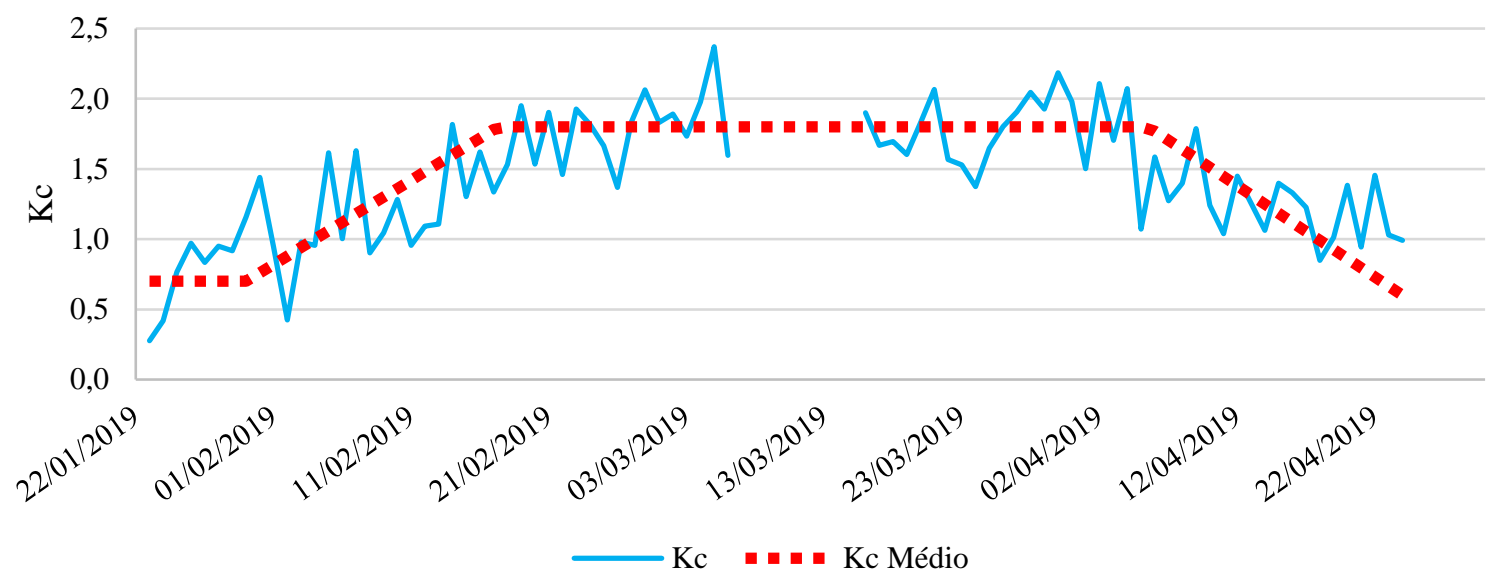

Figura 3. Variações diárias e valores médios dos coeficientes de cultura do sorgo para cada estágio fenológico.

Percebe-se que houve variação dos valores de coeficiente de cultura durante o período experimental. Para o cálculo dos coeficientes de cultivo médios o ciclo da cultura foi dividido em quatro fases ou estádios fenológicos, considerando metodologia proposta por Doorenbos \& Pruitt (1975), da seguinte forma: I) estádio inicial: do plantio até $10 \%$ de cobertura do solo; II) estádio de crescimento: do final da fase inicial até a cobertura vegetal total do solo; III) estádio intermediário: do estabelecimento da cobertura vegetal total do solo até o início da maturação da cultura e IV) estádio final: colheita ou senescência.

A partir dos valores diários da evapotranspiração de cultura e da evapotranspiração de referência foi possível obter valores para cada estádio fenológico da cultura (Tabela 1).

Tabela 1. Duração em dias, e valores de $\mathrm{ET}_{\mathrm{C}}, \mathrm{ET}_{\mathrm{O}}$ e $\mathrm{K}_{\mathrm{C}}$ para cada estádio da cultura do sorgo.

\begin{tabular}{ccccc}
\hline Estádios fenológicos & Duração (dias) & ETc $(\mathrm{mm})$ & $\mathrm{ET}_{\mathrm{O}}(\mathrm{mm})$ & $\mathrm{K}_{\mathrm{C}}$ \\
\hline I & 7 & 27,70 & 39,21 & 0,7 \\
II & 20 & 106,20 & 92,69 & 1,2 \\
III & 45 & 267,58 & 149,30 & 1,8 \\
IV & 20 & 91,4 & 74,4 & 1,2 \\
\hline Total & 92 & 492,85 & 355,60 & -
\end{tabular}

I - Inicial; II - Desenvolvimento vegetativo; III - Floração/Frutificação e IV - Maturação.

Os estádios I, II, III e IV tiveram duração de $7,20,45$ e 20 dias, respectivamente, totalizando 92 dias para o ciclo da cultura. Os valores da $\mathrm{ET}_{\mathrm{C}}$ para as fases inicial, desenvolvimento, floração e maturação foram 27,$70 ; \quad 106,20 ; \quad 267,58$ e $91,4 \mathrm{~mm}$ respectivamente. A demanda total de água para o sorgo no período foi de $492,85 \mathrm{~mm}$. Em experimento realizado por Oliveira et al. (2014) foi encontrado um $\mathrm{ET}_{\mathrm{C}}$ total de $485,45 \mathrm{~mm}$ para a cultura do sorgo irrigado em Ipanguaçú/RN. Enquanto Fonsêca Neto (2014) obteve 499,89 $\mathrm{mm}$, semelhante aos valores obtidos no presente trabalho.

No estádio I ocorre o menor consumo de água pela planta $(0,7)$, sendo ocasionado pela evaporação de água do solo. No II o coeficiente de cultura aumenta $(1,2)$, por conta do desenvolvimento vegetativo da planta. No 
estádio III foi obtido o maior valor médio de $\mathrm{K}_{\mathrm{C}}$ $(1,8)$ o que ocorreu pelo fato de ser um momento em que a planta mais utiliza água, por conta do desenvolvimento de grãos. No quarto estádio há um decréscimo do valor $(1,2)$ quando se comparado ao anterior, pois o ciclo da planta está se encerrando.

Valores semelhantes aos do presente experimento foram obtidos por Oliveira et al. (2014), que para o estádio inicial obtiveram um $\mathrm{K}_{\mathrm{C}}$ de 0,77 , no desenvolvimento vegetativo de 0,93, na floração/frutificação de 1,36 e na maturação de 1,06.

\section{CONCLUSÕES}

De acordo com os resultados obtidos foi possível verificar que a evapotranspiração de referência obtida para todo o período experimental, nesta região, foi de $355,6 \mathrm{~mm}$. A evapotranspiração total da cultura do sorgo forrageiro (SF 15) foi de 492,9 $\mathrm{mm}$ para um ciclo de 92 dias. Os coeficientes de cultivo obtidos para os diferentes estádios fenológicos da cultura foram de 0,7 (Inicial), 1,2 (Crescimento), 1,8 (Floração) e 1,2 (Maturação).

\section{REFERÊNCIAS BIBLIOGRÁFICAS}

ALLEN, R.G. REF-ET: reference evapotranspiration calculator, Version 2.1. Idaho: Idaho University, 2000. 82p.

ALLEN, R. G.; PEREIRA, L. S.; RAES, D.; SMITH, M. Crop evapotranspiration: guidelines for computing crop water requirements. (FAO. Irrigation and Drainage Paper, 56). Roma: FAO, 300p. 1998.

ALVES, E. DA S., LIMA, D. F., BARRETO, J. A. S., DOS SANTOS, D. P., DOS SANTOS, M. A. L. Determinação do coeficiente de cultivo para a cultura do rabanete através de lisimetria de drenagem. Irriga, v. 22, n. 1, p. 194-203, 2017.
AMORIM, D. S., DE SOUSA CARNEIRO, M. S., DO NASCIMENTO ROMILDA, R., DA SILVA, A. L. Pré-secado: uma alternativa para aumentar a segurança alimentar dos rebanhos no período de escassez de forragem. Revista Electrónica de Veterinaria, v. 18, n. 11, p. 113, 2017.

CONCEIÇÃO, C. G. da., PARIZI, A. R. C., SOARES, F. C., DA CONCEIÇÃO, J. A., DA SILVA, G. U., ESSI, R. Coeficientes de cultura (Kc) do feijoeiro comum irrigado. Revista Brasileira de Agricultura Irrigada, v. 10, n. 3, p. 614-620, 2016.

DANTAS, G. D. F., FARIA, R. T. D., SANTOS, G. O., DALRI, A. B., PALARETTI, L. F. Produtividade e qualidade da brachiaria irrigada no outono/inverno. Engenharia Agrícola, v. 36, n. 3, p. 469-481, 2016.

DOORENBOS, J., PRUITT, W. O. Crop water requirement's: Revised. Irrigation and Drainage Paper, 24. Roma: FAO, 144 p. 1975.

GARCIA, A. D. B., VIEIRA, G. H. S., PETERLE, G., BONADIMAN, P. A., JUNIOR, J. L. F., GRAZZIOTI, R. M., LO MONACO, P. A. V., HADDADE, I. R., NETO, A. C. Options for Reference Evapotranspiration Estimation in Northern Espírito Santo and Eastern Minas Gerais States, Brazil. Journal of Experimental Agriculture International, p. $1-14,2019$.

GIRARDI, L. B., PEITER, M. X., BELLÉ, R. A., ROBAINA, A. D., TORRES, R. R., KIRCHNER, J. H., BEN, L. H. B. Evapotranspiração e coeficiente de cultura da alstroemeria (Alstroemeria $\mathrm{x}$ hybrida) cultivada em estufa. Irriga, v. 21, n. 4, p. 817-829, 2016.

KOBAYASHI, B. F., VALERIANO, T. T. B., OLIVEIRA, A. F., SANTANA, M. J. Estimativa da evapotranspiração da cultura do trigo cultivado em Uberaba, MG. Revista Brasileira de Agricultura Irrigada, v. 12, n. 5, p. 2923-2931, 2018. 
MARQUES, P. A. A., DA ROCHA, H. S., JÚNIOR, E. F. F., SOARES, D. A., DUARTE, S. N. Consumo hídrico do manjericão por meio de lisímetro de drenagem. Irriga, v. 20, n. 4, p. 745-761, 2015.

MEHTA, R., PANDEY, V. Reference evapotranspiration (ETo) and crop water requirement $(\mathrm{ETc})$ of wheat and maize in Gujarat. Journal of agrometeorology, v. 17, n. 1, p. 107, 2015.

FONSÊCA NETO, J. Evapotranspiração, crescimento e produção do sorgo em cultivo irrigado na região do baixo açu-RN. 2014. 87 f. Tese (Mestrado em Irrigação e Drenagem) Universidade Federal Rural do Semiárido. Mossoró, Rio Grande do Norte.

OLIVEIRA A. F. M., SILVA J. B. R., SOBRINHO J. E., DE MEDEIROS J. F., NETO J. F. Necessidades hídricas e desenvolvimento do sorgo nas condições do Semiárido brasileiro. In: II Inovagri International Meeting, 2014, Fortaleza. Anais. Fortaleza, 7 p. 2014.

OLIVEIRA, V. S., DA SILVA MORAIS, J. A., FAGUNDES, J. L., DOS SANTOS SANTANA, J. C., LIMA, I. G. S., SANTOS, C. B. Produção e composição químico-bromatológica de gramíneas tropicais submetidas a dois níveis de irrigação. Archives of veterinary science, v. 20, n. 2, 2015.

QUEIROZ, V. A. V., DA SILVA, C. S., DE MENEZES, C. B., SCHAFFERT, R. E., GUIMARÃES, F. F. GUIMARÃES, L. J. M., TARDIN, F. D. Nutritional composition of sorghum [sorghum bicolor (L.) Moench] genotypes cultivated without and with water stress. Journal of Cereal Science, v. 65, p. 103-111, 2015. 